Article

\title{
Hybridization vs. Bond Stretching Isomerism in Ru(II) Cyclometalated Complexes of 2-Phenylpyridine
}

\section{Bertha Molina ${ }^{1}$, Larissa Alexandrova ${ }^{2}$, Ronan LeLagadec ${ }^{3}$, Luis E. Sansores ${ }^{2}$, David Rios-Jara ${ }^{4}$, Francisco Espinosa-Magaña ${ }^{5}$ and Roberto Salcedo ${ }^{2, *}$}

1 Facultad de Ciencias, Universidad Nacional Autónoma de México, Circuito Exterior s/n, Ciudad Universitaria, Coyoacán, Apdo. Post. 70-646, 04510 México D.F., Mexico

2 Instituto de Investigaciones en Materiales, Universidad Nacional Autónoma de México, Circuito Exterior s/n, Ciudad Universitaria, Coyoacán 04510 México D.F., Mexico

3 Instituto de Química, Universidad Nacional Autónoma de México, Circuito Exterior s/n, Ciudad Universitaria, Coyoacán 04510 México D.F., Mexico

4 Instituto Potosino de Investigación Científica y Tecnológica A.C., Camino a la presa de San José 2055, San Luis Potosí, SLP 78216, Mexico

5 Centro de Investigaciones en Materiales Avanzados, SC, Miguel Angel de Cervantes 120, Chihuahua, Chi. 31109, Mexico

* Author to whom correspondence should be adsdressed; E-Mail: salcedo@unam.mx.

Received: 11 October 2011; in revised form: 10 December 2011 / Accepted: 13 December 2011 / Published: 22 December 2011

\begin{abstract}
The phenomenon of formation of two isomers, yellow and orange, of the cyclometalated $\mathrm{Ru}(\mathrm{II})$ complex, $\left[\mathrm{Ru}\left(o-\mathrm{C}_{6} \mathrm{H}_{4}-\mathrm{py}\right)(\mathrm{MeCN})_{4}\right]^{+}$, was investigated by EELS spectroscopy and theoretical calculations. Both forms show very similar structures and spectroscopic properties, but slight differences in X-ray data and absorption between them were noted. No double minimum on the potential energy surface was found and thus these two forms cannot be considered as bond stretching isomers. However, the DFT study revealed the change in the hybridization of the carbon in trans-position of one of acetonitrile ligands. This effect can be responsible for the difference in colour. The results of the theoretical modelling coincide well with the experimental EELS data.
\end{abstract}

Keywords: hybridization isomerism; bond stretch isomerism; $\mathrm{Ru}$ organometallic complexes; trans effect; EELS spectroscopy; time dependent DFT calculations 


\section{Introduction}

The concept of "bond stretch isomerism" was first introduced by Hoffmann based on the theoretical simulation of strained organic tricyclic molecules and was shortly followed by theoretical analyses of other systems [1]. The concept implies an existence of two stable conformations, which are different only by the length of one or more chemical bonds [1,2]. However it took more than a decade to find experimental evidence in support of this very rare kind of isomerism. The early attempts to isolate and to characterize bond-stretch isomers were problematic and many of them were considered as crystallographic artifacts [2-4]. Even nowadays the number of compounds which demonstrate this isomerism is very limited. Thus, according to Rohmer and Bénard [4], the first stretch isomers that had been isolated and fully characterized by Niecke et al. [5] were the biradical 1,3-diphosphacyclobutane2,4-diyl and its corresponding bicyclic form. Later Bertrand et al. [6,7] reported the co-existence in solution of two bond-stretch isomers with trans-annular $\pi$ bonding overlap. The scope of data on the stretching bonds in biradicals has been recently summarized by Breher $[8,9]$ in a very nice review.

Probably the most numerous examples of these isomers have been reported for transition metal complexes. This arises from the large variety of possible structures which may be synthesized for one metal centre using even the same ligands. Many factors such as a small difference between low lying empty molecular orbitals of metal and high lying occupied orbitals of ligand, trans effect, crystallization conditions, etc., can all affect the resulting crystal packing.

Thus, structural variability caused by deviation in bond length was found in the dinuclear ruthenium compound, [(Cp* RuCl $\left.)_{2}(\mu-\mathrm{Cl})_{2}\right]$ [10,11], in trinuclear cobalt [4,12-14] and chromium complexes [15]. Recently, two conformations of a dinuclear iron complex differing in valence angles and bond length were reported by Hammann et al. [16]. The possibility of stretch isomerism in the trinuclear molybdenum compound $\left[\mathrm{Mo}_{3} \mathrm{X}_{12}\right]^{3-}(\mathrm{X}=\mathrm{F}, \mathrm{Cl}, \mathrm{Br}, \mathrm{I})$, was explored by Cavigliasso and Stranger [17]. Additionally, Comba et al. [18] described the distortional isomerism observed in copper(I) complexes of 3,7-diazabicyclo[3.3.1] nonane derivatives.

As can be seen, the grand majority of the complexes explored for the bond-stretch isomerism are many nuclear (di- or tri-) compounds and the biggest variation in distance between atoms was always observed in the metal framework. The difference in metal-metal distance varied approximately between more than $1 \AA$ and several tenths of an $\AA$, meanwhile the variations in bond length of ligands or metal-ligand were much smaller. The strict definition of bond-stretch isomerism implies the existence of a double minimum on the potential energy surface of an investigated compound associated with a specific spin multiplicity [2,19]. Therefore there is a very faint border between bond-stretch and spin-state isomerism for metal complexes, particularly in non-mononuclear ones, where variation in metal-metal distance may result in a change in multiplicity. The thorough theoretical analysis together with accurate experimental measurements is required for determination of this isomerism.

Hybridization isomerism is a very rare concept [20,21], there are few examples of this strange phenomenon, whose definition is "isomerism caused by the change in hybridization on one of the atoms in a pair of species". It has been cited by Collman [20] in NO coordination complexes and for the same reason of the limited cases there are no reviews or deep discussions about it, however the phenomenon plays an important role in the present research. 
The cyclometalated ruthenium(II) compound, $\left[\mathrm{Ru}\left(o-\mathrm{C}_{6} \mathrm{H}_{4}-\mathrm{py}\right)(\mathrm{MeCN})_{4}\right]^{+}$(see Figure 1) analyzed in this work was successfully used in ligand exchange reactions because of its labile $\mathrm{MeCN}$ ligands. It reacts readily with 1,10-phenanthroline (phen) in $\mathrm{MeCN}$ to afford $\left[\mathrm{Ru}\left(o-\mathrm{C}_{6} \mathrm{H}_{4} \text {-py)(phen) }(\mathrm{MeCN})_{2}\right]^{+}\right.$ but the same reaction with 2,2'-bipyridine (bpy) resulted in a significant colour change from yellow to brownish orange, but without any notable structural changes [22,23]. The new orange compound turned out to be slightly more stable than the yellow one and additionally a small difference between them was also observed by UV-Vis spectroscopy. In the first communication on the subject the presence of two structures was suggested [24], but an explanation for this strange phenomenon was not provided since more studies were deemed necessary. Detailed analysis of the X-ray data revealed slight difference in the several bond lengths between the yellow and orange compounds. In the present article the two structures were characterized by electron energy-loss spectroscopy (EELS) in an attempt to find out more distinguished features between them. EELS is a powerful analytical technique that can give information about composition and optical properties of materials [25], where the difference between the yellow and orange forms is very notable. We also intend to further investigate, by means of DFT calculations, a hypothesis whether these two forms may be considered as bond stretch isomers or are a results of a change in the hybridization.

Figure 1. Structure of $\left[\mathrm{Ru}\left(o-\mathrm{C}_{6} \mathrm{H}_{4}-\mathrm{py}\right)(\mathrm{MeCN})_{4}\right]^{+}$, the compound under the study.

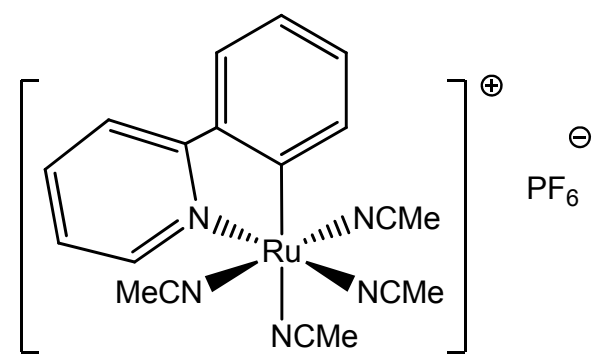

\section{Results and Discussion}

The large similarity of the X-ray data of both isomers of this complex (denoted as yellow and orange) has been established before [24]. However, a more careful examination of the bond lengths in the interactions between $\mathrm{N}$ atom and $\mathrm{C}$ atom in the nitrile ligands shows discrepancies in the corresponding values (Figure 2). These discrepancies are large enough to suggest that the origin of the phenomenon should be bond-stretching isomerism. With this in mind EELS studies were carried out on both isomers.

\subsection{EELS Results}

EELS is an excellent complementary method to X-ray analysis since it allows the measurement of precise elemental composition, and information on the nature of the chemical bonds in the sample [25]. The interactions of fast electrons with the specimen result in excitations of electrons into unoccupied energy levels in the conduction band as well as collective excitations of valence electrons. When a spectrum is obtained by analyzing the energy lost by the incident electrons, the region up to an energy loss of $\approx 50 \mathrm{eV}$ is dominated by collective excitations of valence electrons (plasmon) and by interband 
transitions. At higher energy losses, ionization edges occur due to excitation of core electrons into the conduction band, providing a method for studying the unoccupied conduction states in a solid.

Figure 2. Fragment of the compound in the equatorial plane and relevant distances.

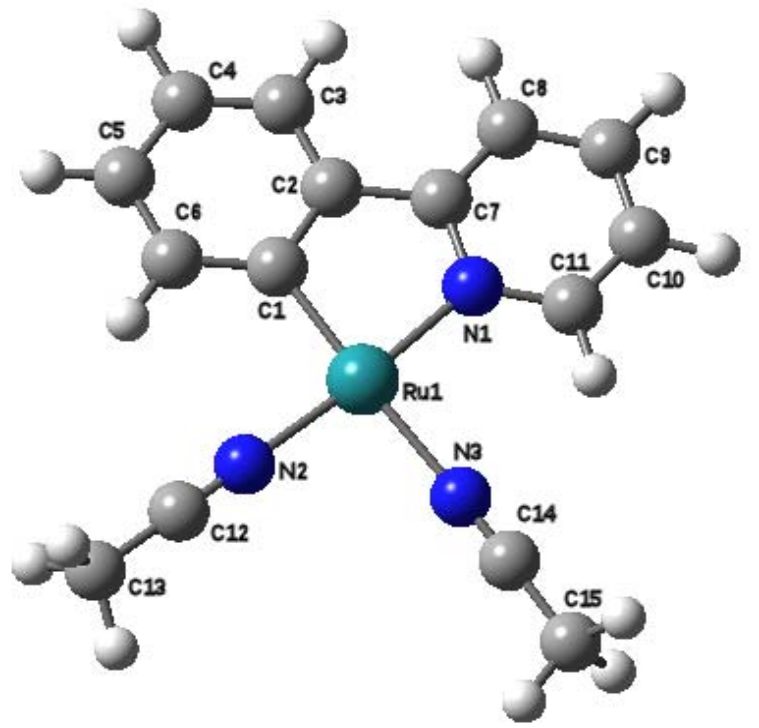

\begin{tabular}{cccc}
\hline $\begin{array}{c}\text { Relevant } \\
\text { Measurement }\end{array}$ & $\begin{array}{c}\text { Yellow } \\
\text { Exp. }\end{array}$ & $\begin{array}{c}\text { Orange } \\
\text { Exp. }\end{array}$ & $\begin{array}{c}\text { Opt. } \\
\text { Structure }\end{array}$ \\
\hline N2-C12 & 1.136 & 1.117 & 1.173 \\
C12-C13 & 1.444 & 1.458 & 1.457 \\
C14-C15 & 1.446 & 1.425 & 1.458 \\
Ru1-N2 & 2.054 & 2.046 & 2.027 \\
Ru1-N3 & 2.152 & 2.146 & 2.223 \\
Ru1-C1 & 2.014 & 2.014 & 2.038 \\
Ru1-N2-C12-C13 & 25.0 & 22.67 & 35.35 \\
\hline
\end{tabular}

These core-level processes are mostly sensitive to final states since the initial states have narrow energy widths. Besides the well defined ionization edges, there is a fine structure superposed on the edge and extending up to about $50 \mathrm{eV}$ from the edge onset, which is associated with the density of unoccupied states in the conduction band, known as the Energy Loss Near Edge Structure (ELNES).

Generally, for a particular elemental ionization edge, the observed ELNES exhibits a structure that is specific to the arrangement, as well as the type of atoms within the first coordination shell. Since the inner-shell excitation process is highly localized on a particular atomic site, if two different coordinations of one element co-exist within the same structure, then the individual contributions of the different sites to the observed ELNES is a simple linear sum weighted by the respective site occupancies. Consequently, this technique has been used in the determination of $\mathrm{sp}^{2} / \mathrm{sp}^{3}$ bonded carbon ratios in diamond-like films [26].

Besides the Density of States (DOS) shape of the ELNES, any excess energy from the ionization energy can be thought as a wave emanating from the ionized atom. If this wave has only a few $\mathrm{eV}$, it undergoes plural elastic scattering from the surrounding atoms, appearing as a broad signal some 20-40 eV above the edge onset. This is known as multiple scattering resonance (MSR), arising from a resonant scattering event involving the excited electron and a particular shell of atoms. The energies of 
these features above the edge onset have been shown to be proportional to $1 / \mathrm{r}^{2}$, where $\mathrm{r}$ is the bond length from the ionized atom.

Figure 3 shows the energy loss function for the yellow and orange samples. The dominant feature in the energy loss spectra is the volume plasmon, showing a well-defined maximum at $22.6 \mathrm{eV}$ for both samples. The maximum in the energy loss function is the plasmon energy that can be written in a first approximation as:

$$
E_{p}^{2}=\frac{\hbar^{2} n e^{2}}{\varepsilon_{0} m}
$$

where, $\mathrm{n}$ is the total charge density, e the electron charge, $\mathrm{m}$ the mass of the electron and $\varepsilon_{0}$ the permittivity of vacuum [27]. From Figure 3, we can assert that the electronic density is the same for both samples.

Figure 3. Energy loss function, $\operatorname{Im}(-1 / \varepsilon)$, for yellow (continuous) and orange (dotted) samples, from the low energy loss region of EELS spectra.

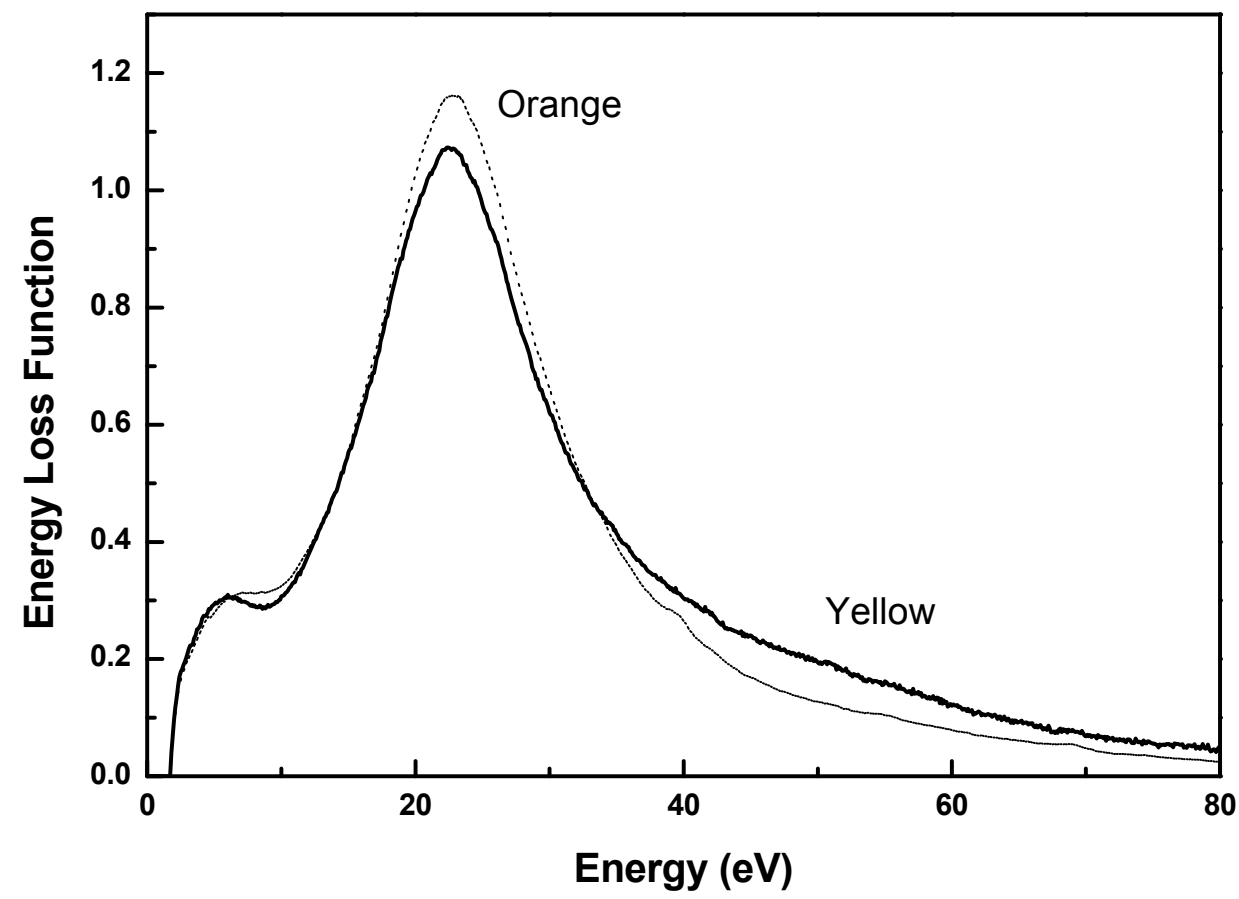

Figure 4 shows energy-loss spectra in the high-energy region for both samples, where carbon (C) and nitrogen $(\mathrm{N}) \mathrm{K}$ edge as well as the ruthenium $(\mathrm{Ru}) \mathrm{M}_{3}$ ionization edge are clearly observed. Here the spectra have been normalized so that post edges above $\mathrm{Ru}$ ionization edge coincide. Spectra were background-subtracted and Fourier-ratio deconvoluted, to eliminate multiple scattering effects. Quantitative elemental analysis has been carried out by the usual Egerton method [27]. Elemental ratios obtained are: $\mathrm{N} / \mathrm{C}=0.23 \pm 0.04$ for yellow and $\mathrm{N} / \mathrm{C}=0.26 \pm 0.04$ for orange. $\mathrm{Ru} / \mathrm{C}$ ratios are also very similar for both samples. Therefore, the difference in colour couldn't be explained by variation in the composition. 
Figure 4. High-energy loss region of EELS spectra showing the $\mathrm{K}$ ionization edges from $\mathrm{C}$ and $\mathrm{N}$ and $\mathrm{Ru} \mathrm{M}_{3}$ edge of yellow (continuous) and orange (dotted) samples.

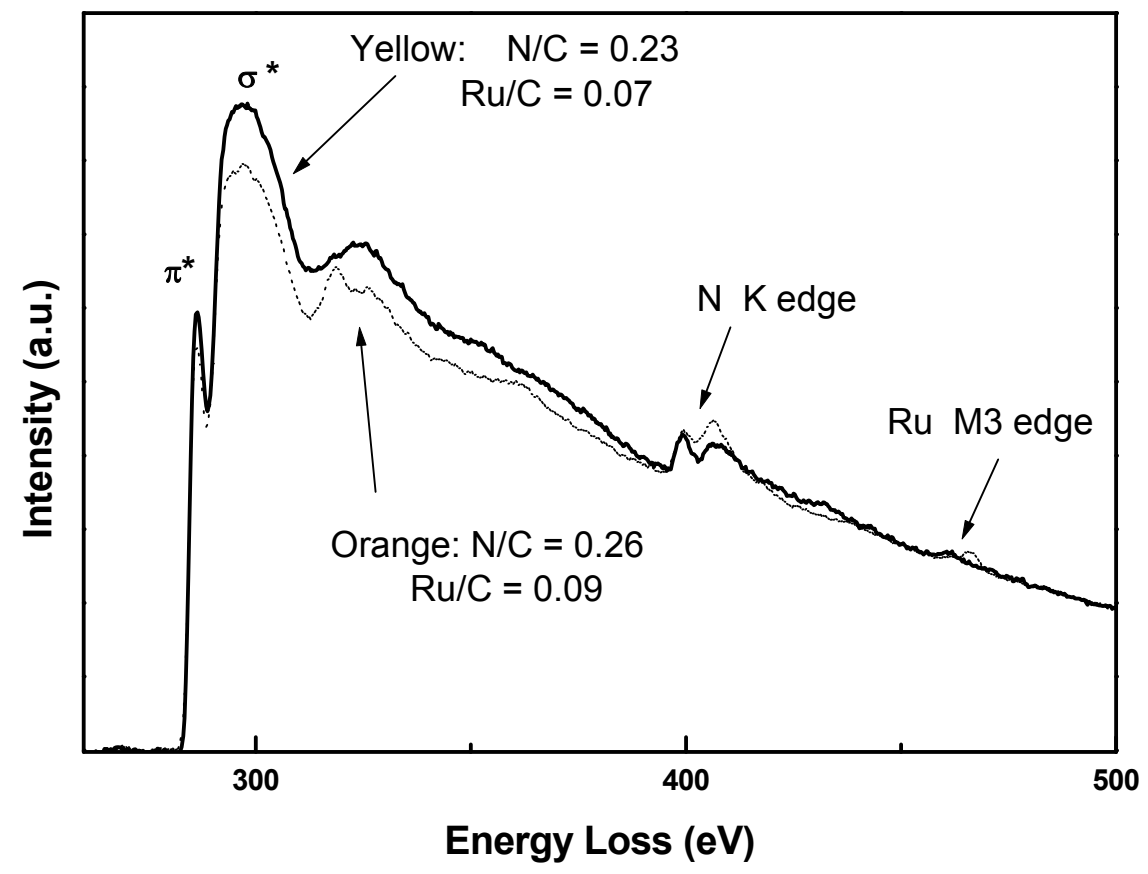

Figure 5 shows energy-loss spectra for carbon $\mathrm{K}$ ionization edge for both samples, where spectra were shifted up for clarity. The ratios of $\mathrm{sp}^{2} / \mathrm{sp}^{3}$ obtained by fitting Lorentzian curves to $\pi^{*}$ and $\sigma^{*}$ peaks in the studied samples and the spectrum from pure graphite (not shown in the figure) are $\mathrm{R}_{\mathrm{y}}=83.7 \%$ and $\mathrm{R}_{\mathrm{o}}=95.1 \%$ respectively indicating a change in the hybridization of the isomers.

Figure 5. Carbon K Ionization edge, showing $1 s-\pi^{*}$ and $1 s-\sigma^{*}$ peaks. Signaled energies correspond to MSR peaks in yellow (continuous) and orange (dotted) samples.

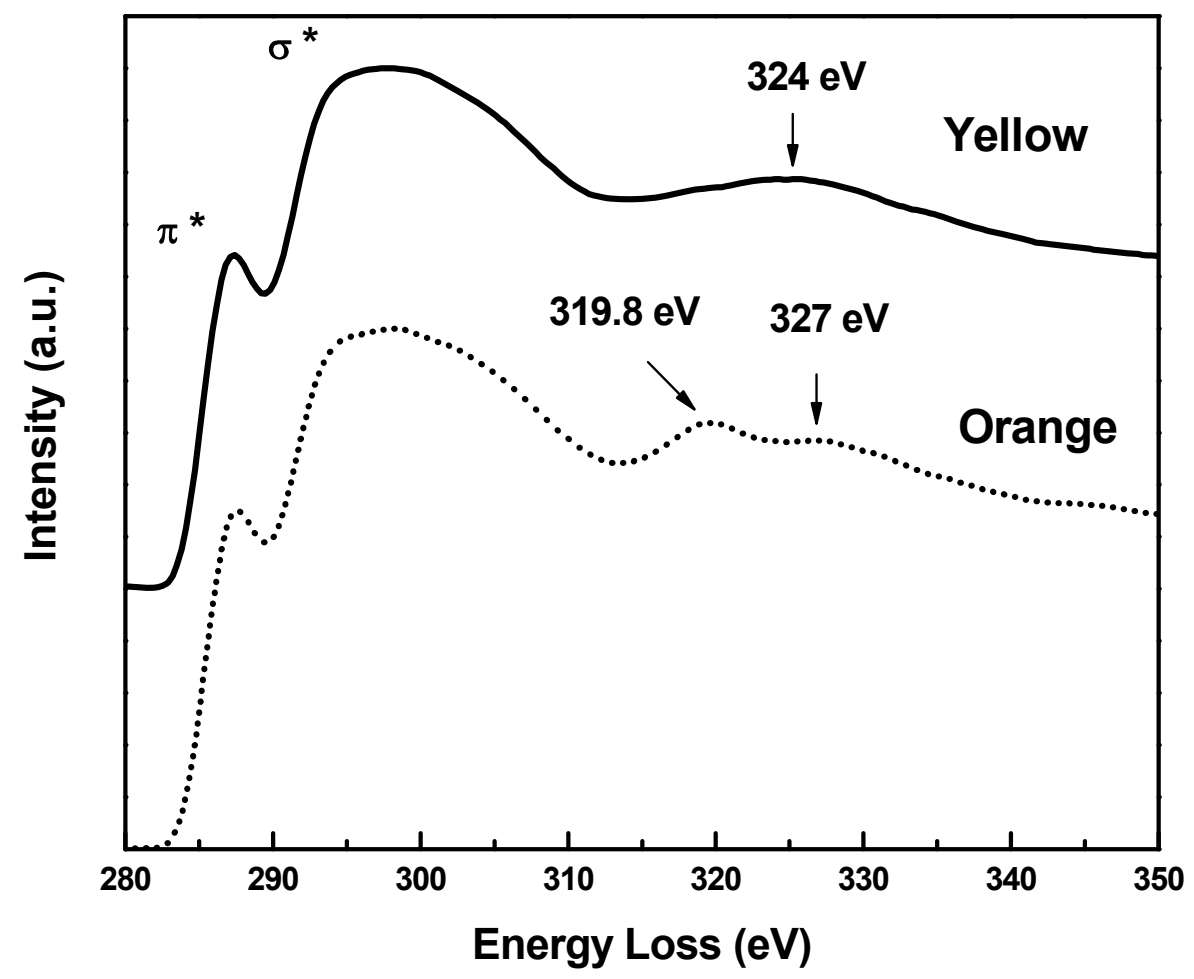


Figure 6 shows the energy-loss spectra for nitrogen $\mathrm{K}$ ionization edge for both samples, where spectra were displaced upwards for clarity. It is evident that the post edge fine structure has changed. The Multiple Scattering Resonance peak in the orange sample has moved to the right of the yellow sample, indicating that bond lengths in the orange isomer are smaller than in the yellow one.

Figure 6. Nitrogen $\mathrm{K}$ ionization edge, showing $\pi^{*}, \sigma^{*}$ and multiple scattering resonance signal in yellow (continuous) and orange (dotted) samples.

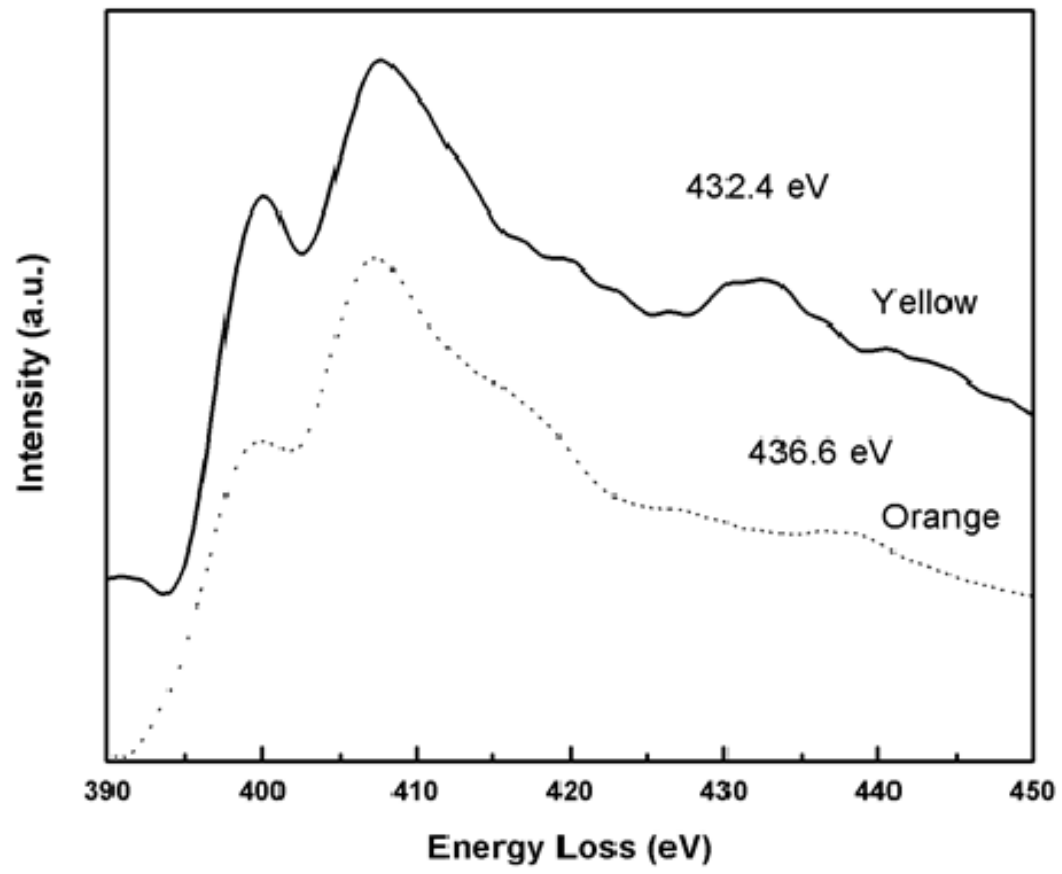

We can make a semi-quantitative analysis on relative changes of bond-lengths in the yellow and orange samples. We can estimate the relative $\mathrm{C}-\mathrm{N}$ bond-length variation between both samples by applying the empirical Equation [28]:

$$
\Delta \mathrm{ER}^{2}=\text { constant }
$$

where, $\Delta \mathrm{E}$ is the energy of the Multiple Scattering Resonant above the ionization threshold and $\mathrm{R}$ is the bond-length.

Equation (1) can be written, in our case, as:

$$
\frac{R_{Y}}{R_{O}}=\sqrt{\frac{\Delta E_{O}}{\Delta E_{Y}}}
$$

From Figure 5, we obtain the bond length in the yellow sample to bond length in the orange sample ratios for $C$ atoms: $\mathrm{R}_{\mathrm{Y}} / \mathrm{R}_{\mathrm{O} 1}=1.04$ and $\mathrm{R}_{\mathrm{Y}} / \mathrm{R}_{\mathrm{O} 2}=0.95$, where $\mathrm{R}_{\mathrm{O} 1}$ and $\mathrm{R}_{\mathrm{O} 2}$ are related to the MSR peaks at $327 \mathrm{eV}$ and $319.8 \mathrm{eV}$ respectively. From the $\mathrm{N}$ post-edge structure in Figure 6 we have $\mathrm{R}_{\mathrm{Y}} / \mathrm{R}_{\mathrm{O}}=1.04$.

These results are qualitatively in good agreement with values obtained from X-ray analysis (see Figure 2). For $\mathrm{C}$ atoms, $\mathrm{X}$-rays show that some bond lengths in yellow sample are increased with respect to the orange sample, as well as the opposite effect, e.g., in C12-C13 the calculated ratio is 0.990 and in $\mathrm{C} 14-\mathrm{C} 15$ the ratio is 1.014. On the other hand, for $\mathrm{N}$ atoms the bond lengths ratios are always greater than one (within the estimated uncertainty). In this case, the maximum change occurs in 
N2-C12 bond, the ratio being 1.03. Other minor bond stretching implies a minor shift in MSR peak positions and would be superposed on the main peak in EELS spectra.

\subsection{Computational Results}

Since our X-ray and EELS results point out the N2-C12 bond as that in which the maximum change occurred, we grossly estimated the interaction energy $\left(E_{\text {int }}\right)$ between the fragments $A$ and $B$ within the total molecule; the fragments $\mathrm{A}$ and $\mathrm{B}$ in both compounds, yellow and orange, were defined by the breaking of the N2-C12 bond (Figures 1 and 2). To calculate the interaction energy in both cases, we carried out the single point energy calculations of each fragment $\left(E_{A}\right.$ and $\left.E_{B}\right)$, and the single point energy calculations of the total molecule $\left(E_{t}\right)$. The $E_{\text {int }}$ energy was obtained using the equation: $E_{\text {int }}=E_{t}-\left(E_{A}+E_{B}\right)$.

Comparing the $\mathrm{E}_{\text {int }}$ energy calculated for the yellow and orange molecules, we obtained a difference between them in the order of van der Waals bond $(3 \mathrm{kcal} / \mathrm{mol})$. The optimized structure was achieved starting from the experimental structures of both the yellow and orange compounds, in both cases we reached the same optimized geometry. The relevant measurements for this species are shown in Figure 2. It is worth noting that there are important differences among the three structures, which will be discussed below. The important point is that these differences allow finding the source of the strange isomers.

Additionally, the TDDFT calculations performed in acetonitrile environment (see Table 1) are in good agreement with the experimental UV-Vis spectra. The experimental solution spectra were very similar: The same absorption maximums observed at $\lambda=240,288$ and $375 \mathrm{~nm}$ for both compounds and the only difference was noted in values of the extinction coefficients. The extinction coefficient of longer wavelength absorption of the orange compound was slightly (about 5-13\% depending on the solvent) higher than that of the yellow sample. Interestingly that this difference in MeCN solution spectra was less than in the spectra run in other solutions such as $\mathrm{CH}_{2} \mathrm{Cl}_{2}$ and $\mathrm{MeOH}$ [24].

Table 1. TDDFT exited states spectra performed in MeNC environment. Main contribution (MC) to each exited state with the HOMO corresponding to the 92 orbital. Excitation energies $(\mathrm{EE}, \mathrm{eV})$, wave lengths $(\lambda, \mathrm{nm})$, percentage of probability $(\% \mathrm{P})$ that the transition occurs and oscillator strengths (f) computed for both yellow and orange isomers.

\begin{tabular}{|ccccc|ccccc|}
\hline \multicolumn{5}{c|}{ Yellow compound } & \multicolumn{5}{c|}{ Orange compound } \\
\hline $\mathbf{M C}$ & $\mathbf{\% P}$ & $\mathbf{E E}$ & $\boldsymbol{\lambda}$ & $\mathbf{f}$ & $\mathbf{M C}$ & $\mathbf{\% P}$ & $\mathbf{E E}$ & $\boldsymbol{\lambda}$ & $\mathbf{f}$ \\
\hline $\mathrm{S} 92 \rightarrow 93$ & 69.29 & 2.56 & 484 & 0.0016 & $\mathrm{~S} 92 \rightarrow 93$ & 69.04 & 2.57 & 482 & 0.0028 \\
$\mathrm{~S} 91 \rightarrow 93$ & 70.32 & 2.67 & 464 & 0.0002 & $\mathrm{~S} 91 \rightarrow 93$ & 70.28 & 2.68 & 463 & 0.0003 \\
$\mathrm{~S} 92 \rightarrow 94$ & 65.77 & 2.96 & 418 & 0.0023 & $\mathrm{~S} 92 \rightarrow 94$ & 65.34 & 2.99 & 415 & 0.0021 \\
$\mathrm{~S} 91 \rightarrow 94$ & 70.44 & 3.07 & 404 & 0.0002 & $\mathrm{~S} 91 \rightarrow 94$ & 70.23 & 3.10 & 400 & 0.0005 \\
$\mathrm{~S} 90 \rightarrow 93$ & 60.34 & 3.21 & 387 & 0.1327 & $\mathrm{~S} 90 \rightarrow 93$ & 59.56 & 3.19 & 389 & 0.1140 \\
$\mathrm{~S} 90 \rightarrow 94$ & 65.96 & 3.40 & 365 & 0.0219 & $\mathrm{~S} 90 \rightarrow 94$ & 65.36 & 3.45 & 359 & 0.0362 \\
$\mathrm{~S} 89 \rightarrow 93$ & 59.50 & 3.90 & 318 & 0.0678 & $\mathrm{~S} 89 \rightarrow 93$ & 53.64 & 3.99 & 311 & 0.0690 \\
$\mathrm{~S} 92 \rightarrow 95$ & 62.05 & 3.94 & 315 & 0.0013 & $\mathrm{~S} 92 \rightarrow 95$ & 51.16 & 4.01 & 309 & 0.0166 \\
$\mathrm{~S} 92 \rightarrow 96$ & 48.26 & 4.08 & 304 & 0.0227 & $\mathrm{~S} 92 \rightarrow 96$ & 52.78 & 4.12 & 301 & 0.0327 \\
$\mathrm{~S} 88 \rightarrow 93$ & 53.81 & 4.09 & 303 & 0.0104 & $\mathrm{~S} 88 \rightarrow 93$ & 62.60 & 4.13 & 300 & 0.0037 \\
\hline
\end{tabular}


However it was also noted a little more pronounced kind of shoulder around 480-500 nm for the orange sample, but the difference was very difficult to detect numerically. This part of the UV-Vis spectra run in $\mathrm{MeCN}$ solution for the yellow and orange compounds is shown in Figure 7 for clarity.

Figure 7. UV-vis spectra of $\mathrm{MeCN}$ solutions of yellow (dashed line) and orange (solid line) compounds.

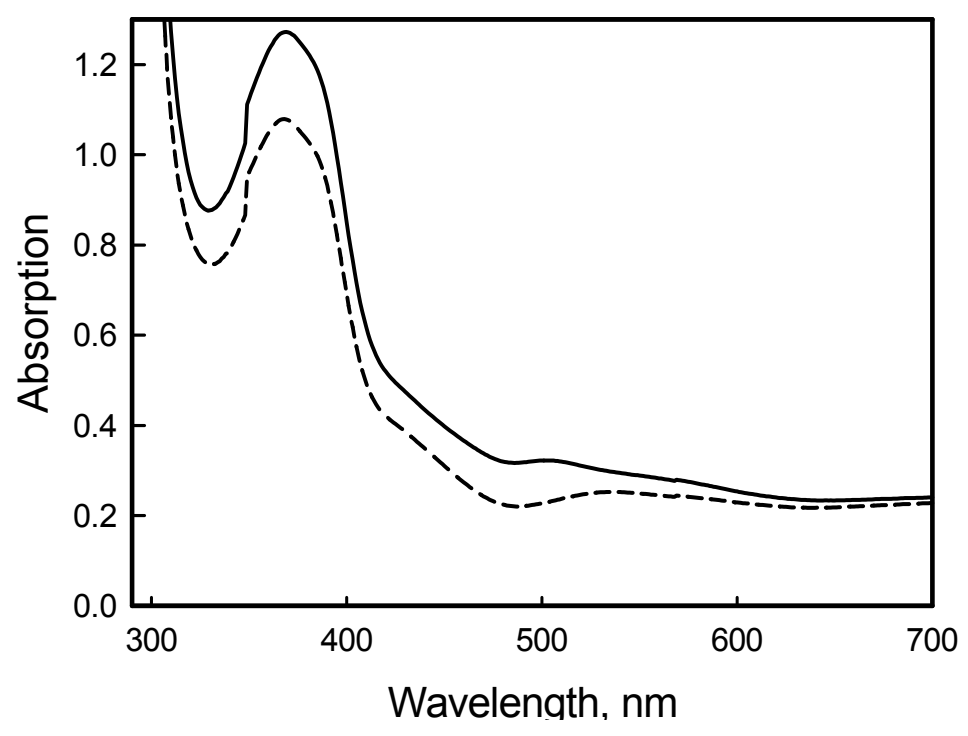

Explicitly, for both the yellow and orange compounds, the TDDFT calculations predict that the vertical transition more strongly absorbed with similar intensities and probabilities ( $f=0.1327$, $\% \mathrm{P}=60.26$; and $\mathrm{f}=0.1140, \% \mathrm{P}=59.45$ respectively) corresponds to the transition HOMO-2 $\rightarrow$ LUMO (3.21 and $3.19 \mathrm{eV}$ ) localized at 387 and $389 \mathrm{~nm}$ respectively. Comparing with the UV-Vis spectra, these theoretical transitions are matching with an experimental wide band observed for both compounds at $374 \mathrm{~nm}$. This absorption band is at near ultra-violet range and does not impact much in colour; an absorption in longer wavelength should be responsible for the colour differences. Our TDDFT calculations show other very similar absorptions at 400-404 and 415-418 nm for both compounds (transitions HOMO-1 $\rightarrow$ LUMO+1 and HOMO $\rightarrow$ LUMO+1). However the biggest difference is observed for the HOMO $\rightarrow$ LUMO vertical transition localized at $480 \mathrm{~nm}$ (484 nm for the yellow and $482 \mathrm{~nm}$ for the orange). It has approximately the same probability for both compounds (69.29\% and $69.04 \%$ respectively), but is $\sim 42 \%$ more strongly absorbed by the orange isomer. This coincides with the experimental observation of more pronounced shoulder in this region for the orange sample (see Figure 7 above).

Nevertheless, no dissimilarities were found when we examined the corresponding HOMO-1, $\mathrm{HOMO}$, LUMO and LUMO+1 of both structures (Figure 8). At this point the most important question is what is the source of these changes. A qualitative analysis of the wave functions may suggest an answer. It seems that the phenomenon occurs due to the presence of the phenyl-pyridine ligand (3), its trans effect and the nature of its frontier orbitals. The HOMO-LUMO gap of free 3 is relatively large $(5.7 \mathrm{eV})$ but the nature of the LUMO is an antibonding $\pi$ orbital. However it is placed so far from the HOMO that a strong interaction between the frontier orbitals is not expected. 
This situation changes dramatically when the fragment 3 forms part of the organometallic compounds, in these cases the HOMO-LUMO gap for both complexes are $2.456 \mathrm{eV}$ for the orange compound and $2.479 \mathrm{eV}$ for the yellow compound. Now the gap is narrow in both cases and it is expected some kind of interaction of both orbitals even more considering that the LUMO is an antibonding molecular orbital very appropriated for backbonding (see Figure 8).

Figure 8. HOMO, LUMO set for yellow isomer.

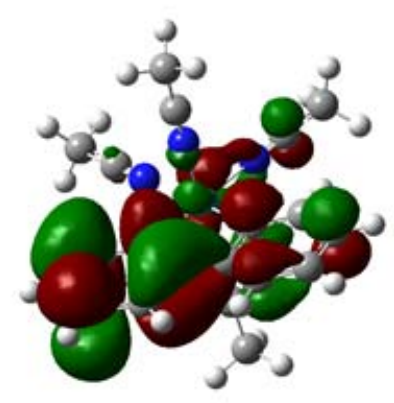

LUMO

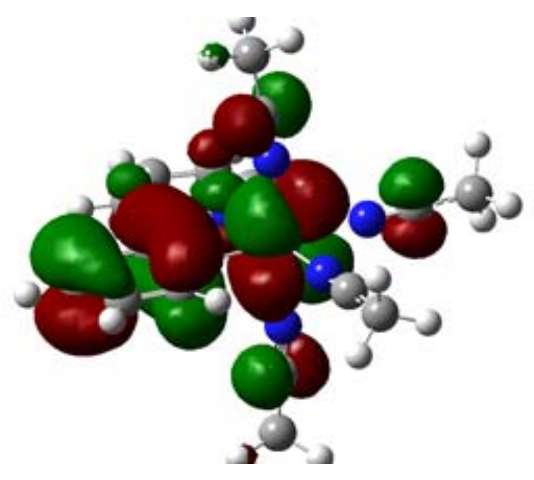

$\mathrm{HOMO}$

Thus, the difference between the gaps is only $0.023 \mathrm{eV}$; therefore in an idealized situation the compound would present two local minima corresponding to the structures, which impose different effect on the acetonitriles in the trans-position with respect to the phpy ligand. Indeed, the N2-C12 bond length, that belongs to the fragment of acetonitrile in the position trans to the nitrogen atom of phpy, is $1.117 \AA$ for the orange compound; whereas the same bond in the yellow sample is $1.137 \AA$ due to the difference in energy caused by the variation in the backbonding. This difference is not enough to describe these two forms as bond stretch isomers, but it may suggest an exchange hybridization case. This can be verified by analyzing the dihedral angles between Ru-N2-C12-C13 (Figure 2) which have the values of $22.67^{\circ}$ for the orange and $25.0^{\circ}$ for the yellow compound. This assumption is in agreement with the EELS result that the difference between both compounds is the $\mathrm{sp} 2 / \mathrm{sp} 3$ ratio. The transition between both different hybridization structures was computed, but no definite transition state was found. The failure to find a double minimum on the potential energy curve has been reported for various transition metal complexes searched for bond stretch isomerism $[12,18]$. Some authors proposed existence of fluxionallity between both structures in solution, which can be trapped by crystallization. However the hypothesis of simple thermo-fluctuations in solution can't be a 
plausible explanation for the phenomenon of the yellow and orange $\mathrm{Ru}(\mathrm{II})$ compounds. The transformation of the yellow form into the orange one or vice versa has never occurred in any kind of solvent, even at elevated temperatures. Thus, absence of the definite transition between these two forms demonstrated by the modelling is confirmed by the experimental observations. The yellow compound converts into the orange form only in the presence of pyridine or bipyridine molecules. These molecules in an attempt of ligand substitution reaction somehow cause the fluctuation in relatively labile acetonitrile ligands that results in the colour change. Thus it may be a case of "induced" fluxionality.

\section{Experimental}

\subsection{Synthesis of the Ruthenium(II) Complexes}

The yellow and orange forms of the ruthenium complex, $\left[\mathrm{Ru}\left(o-\mathrm{C}_{6} \mathrm{H}_{4}-\mathrm{py}\right)(\mathrm{MeCN})_{4}\right]^{+}$, were synthesized as described in reference [24]. Their UV-Vis spectra were obtained in freshly prepared $\mathrm{MeCN}$ solutions using a Varian Cary $400 \mathrm{UV}-\mathrm{Vis}$ spectrophotometer.

\subsection{EELS Spectroscopy}

Electron energy loss spectra were obtained using a Gatan Parallel Electron Energy Loss Spectrometer (PEELS model 766) attached to a Philips CM-200 transmission electron microscope (TEM). Spectra were taken in diffraction mode with $0.3 \mathrm{eV} / \mathrm{ch}$ dispersion, an aperture of $3 \mathrm{~mm}$ and a collection semi-angle of $2.8 \mathrm{mrad}$. The resolution of the spectra was determined by measuring the full width at half-maximum (FWHM) of the zero-loss peak. This was typically close to $1.5 \mathrm{eV}$ for the low energy region, when the TEM was operated at $200 \mathrm{kV}$. The EELS spectra were corrected for dark current and readout noise. The channel-to-channel gain variation was minimized normalizing the experimental spectrum with the gain spectrum of the spectrometer obtained independently. Spectra in the high-energy region were background-subtracted fitting the pre-edge backgrounds with a power-law function and then Fourier-Ratio deconvoluted to remove multiple scattering components from the spectra. In the low-energy region, spectra were Fourier-Log deconvoluted to obtain single scattering distributions $\mathrm{S}(\mathrm{E})$.

\subsection{Molecular Modelling Details}

All calculations were carried out using the BPW91 scheme $[29,30]$ with the $6-31 \mathrm{G} *$ basis, the code used was Gaussian03 [31] on single point calculation performed on the published x-ray structures. TDDFT calculations were carried out at the same level of theory in order to simulate the visible spectra previously reported. The application of TDDFT in the field of quantum chemistry is relatively recent $[32,33]$, and a number of problems have been identified in its implementation. Although the exact functional $E_{x c}$ of DFT is not known, there are a number of approximate forms that give useful results. In the case of TDDFT the situation is more complicated due to the required functional $A_{x c}$ is time dependent on the electron density. The simplest approximation is the so called, Adiabatic Approximation, which uses the zero frequency limit of $A_{x c}$ for treatments of finite frequency perturbations. This is justified for the low-frequency domain. In the high frequency domain some 
overestimation of the excitation energies is observed and certain excitations out of the $\sigma$-system are underestimated. In general, better results are obtained for functional that provide an exchange-correlation potential with correct asymptotic behaviour $(-1 / \mathrm{r})[34,35]$.

\section{Conclusions}

The phenomenon of the formation of two forms distinguished by colour (yellow and dark orange) in the cyclometalated $\mathrm{Ru}(\mathrm{II})$ complex, [Ru(o-C6H4-py)(MeCN)4]+, was studied by means of EELS spectroscopy and DFT calculations. EELS spectroscopy confirmed the same composition of both forms but revealed a small difference in the hybridization states of the carbon atoms and variations in the $\mathrm{C}-\mathrm{C}$ and $\mathrm{C}-\mathrm{N}$ bond lengths of the ligands. Careful analysis of $\mathrm{X}$-ray data also showed minute differences in some bond length of the acetonitrile ligands. The calculated energy surfaces for these two forms demonstrated the existence of only one minimum and thus the yellow and orange compounds could not be considered as a case of bond-stretch isomerism. However a small difference in energy resulting from backbonding between the yellow and orange forms was found. This affects the geometry of the compounds and leads to a change in the hybridization of the carbon in the transacetonitrile ligand. The observed difference in colour is rather caused by slight difference in the hybridization. Thus, the results of theoretical modelling coincide well with the experimental observations.

\section{Acknowledgements}

The authors wish to thank DGTIC-UNAM and Instituto Potosino de Investigación Científica y Tecnológica (IPICYT) for providing computing time and Project No. PAPIIT-IN107807 for funding.

\section{References and Notes}

1. Stohrer, W.D.; Hoffmann, R. The electronic Structure and reactivity of strained tricyclic hydrocarbons. J. Am. Chem. Soc. 1972, 94, 779-786.

2. Parkin, G. Bond-stretch isomerism in transition metal complexes: A reevaluation of crystallographic data. Chem. Rev. 1993, 93, 887-911.

3. Yoon, K.; Parkin, G.; Rheingold, A.L. Bond-stretch isomerism in the chlorooxomolybdenum complexes cis-mer-Moocl2(PR3)3: A reinvestigation. J. Am. Chem. Soc. 1992, 114, 2210-2218.

4. Rohmer, M.M.; Bénard, M. Bond-stretch isomerism in strained inorganic molecules and in transition metal complexes: A revival? Chem. Soc. Rev. 2001, 30, 340-354.

5. Niecke, E.; Fuchs, A.; Nieger, M. Valence isomerization of a 1,3-diphosphacyclobutane-2,4-diyl: Photochemical ring closure to 2,4-diphosphabicyclo[1.1.0]butane and its thermal ring opening to gauche-1,4-diphosphabutadiene. Angew. Chem. Int. Ed. 1999, 38, 3028-3031.

6. Rodriguez, A.; Olsen, R.A.; Ghaderi, N.; Scheschkewitz, D.; Tham, F.S.; Mueller, L.J.; Bertrand, G. Evidence for the coexistence of two bond-stretch isomers in solution. Angew. Chem. Int. Ed. 2004, 43, 4880-4883.

7. Soleilhavoup, M.; Bertrand, G. Let's play with valence isomers: The influence of different main group elements on their relative stability. Bull. Chem. Soc. Jpn. 2007, 80, 1241-1252. 
8. Breher, F. Stretching bonds in main group element compounds-Borderlines between biradicals and closed-shell species. Coord. Chem. Rev. 2007, 251, 1007-1043.

9. Grützmacher, H.; Breher, F. Odd-electrons bonds and biradicals in main group element chemistry. Angew. Chem. Int. Ed. 2002, 41, 4006-4011.

10. McGrady, J.E. [(Cp* RuCl $\left.)_{2}(\mu-\mathrm{Cl})_{2}\right]$ : Bond-stretch or spin-state isomerism?. Angew. Chem. Int. Ed. 2000, 39, 3077-3079.

11. Feng, Q.; Rauchfuss, T.B.; Wilson, S. Geometric isomerism based on metal-metal bonds. J. Am. Chem. Soc. 1995, 117, 4702-4703.

12. Pantazis, D.A.; McGrady, J.E. A three-state model for the polymorphism in linear tricobalt compounds. J. Am. Chem. Soc. 2006, 128, 4128-4135.

13. Clérac, R.; Cotton, F.A.; Daniels, L.M.; Dunbar, K.R.; Murillo, C.A.; Wang, X. Tuning the metal metal bonds in the linear tricobalt compound Co3(Dpa)4C12: Bond-stretch and spin-state isomers. Inorg. Chem. 2001, 40, 1256-1264.

14. Rohmer, M.M.; Strich, A.; Bénard, M.; Malrieu, J.P. Metal-metal bond length variability in $\mathrm{Co}(3)$ (Dipyridylamide)(4)Cl(2): Bond-stretch isomerism, crystal field effects, or spin transition process? A DFT study. J. Am. Chem. Soc. 2001, 123, 9126-9134.

15. Berry, J.F.; Cotton, F.A.; Lu, T.; Murillo, C.A.; Roberts, B.K.; Wang, X. Molecular and electronic structures by design: Tuning symmetrical and unsymmetrical linear trichromium chains. J. Am. Chem. Soc. 2004, 126, 7082-7096.

16. Hammann, B.; Chen, C.; Flörke, U.; Hauptmann, R.; Bill, E.; Sinnecker, S.; Henkel, G. Alphaand beta-[Fe(2)(Mu-Stbu)(2)(Stbu)(4)](2-): Coexistence of two bond-stretch isomers of a classical bitetrahedral metal chalcogenolate compound. Angew. Chem. Int. Ed. 2006, 45, 8245-8249.

17. Cavigliasso, G.; Stranger, R. Electronic structure and metal-metal interactions in trinuclear faceshared [M3X12]3- (M = Mo, W; X = F, Cl, Br, I) systems. Inorg. Chem. 2008, 47, 3072-3083.

18. Born, K.; Comba, P.; Kerscher, M.; Linti, G.; Pritzkow, H.; Rohwer, H. Distortional isomerism with Copper(I) complexes of 3,7-diazabicyclo[3.3.1]nonane derivatives. Dalton Trans. 2009, 362-367.

19. Parkin, G.; Hoffmann, R. Spin-state isomers and bond stretch isomers: A comment on the article "bond-stretch isomers: Fact not fictio". Angew. Chem. Int. Ed. 1994, 33, 1462.

20. Collman, J.P.; Farnham, P.; Dolcetti, G. Intramolecular redox equilibriums of cobalt-nitrosyl complexes. J. Am. Chem. Soc. 1971, 93, 1788-1790.

21. Alam, M.S.; Xi, H.-W.; Lim, K.H. Theoretical studies of germaallenic structure, bonding and hyperconjugation. Organometallics 2009, 28, 3678-3685.

22. Ryabov, A.D.; Sukharev, V.S.; Alexandrova, L.; Le Lagadec, R.; Pfeffer, M. New synthesis and new bio-application of cyclometalated Ruthenium(II) complexes for fast mediated electron transfer with peroxidase and glucose oxidase. Inorg. Chem. 2001, 40, 6529-6532.

23. Ryabov, A.D.; Le Lagadec, R.; Estevez, H.; Toscano, R.A.; Hernández, S.; Alexandrova, L.; Kurova, V.S.; Fischer, A.; Sirlin, C.; Pfeffer, M. Synthesis, characterization, and electrochemistry of biorelevant photosensitive low-potential orthometalated ruthenium complexes. Inorg. Chem. 2005, 44, 1626-1634.

24. Ryabov, A.D.; Estevez, H.; Alexandrova, L.; Pfeffer, M.; Le Lagadec, R. Unusual phenomenon in the chemistry of orthometalated Ruthenium(II) complexes. Inorg. Chim. Acta 2006, 359, 883-887. 
25. Ahn, C.C. Transmission Electron Energy Loss Spectrometry in Materials Science and the EELS Atlas; WILEY-VCH Verlag GmbH \& Co. KgaA: Weinheim, Germany, 2004.

26. Ponsonnet, L.; Donnet, C.; Varlot, K.; Martin, J.M.; Grill, A.; Patel, V. EELS analysis of hydrogenated diamond-like carbon films. Thin Solid Films 1998, 319, 97-100.

27. Egerton, R.F. Electron Energy Loss Spectroscopy in the Electron Microscope; Plenum Press: New York, NY, USA, 1996.

28. Kurata, H.; Lefevre, E.; Colliex, C. Electron-energy-loss near-edge structures in the oxygen K-edge spectra of transition-metal oxides. Phys. Rev. B 1992, 47, 13763-13768.

29. Becke, A.D. Density-functional exchange-energy approximation with correct asymptotic behavior. Phys. Rev. A 1988, 38, 3098-3100.

30. Perdew, J.P.; Wang, Y. Accurate and simple analytic representation of the electron-gas correlation energy. Phys. Rev. B 1992, 45, 13244-13249.

31. Frisch, M.J.; Trucks, G.W.; Schlegel, H.B.; Scuseira, G.E.; Robb, M.A.; Cheeseman, J.R.; Zakrzewski, V.G.; Montgomery, J.A., Jr; Stratmann, R.E.; Burant, J.C.; et al. Gaussian03; Gaussian Inc.: Wallingford, CT, USA, 2004.

32. van Gisbergen, S.J.A.; Snijders, J.G.; Baerends, E.J. A Density functional theory study of frequency-dependent polarizabilities and van der waals dispersion coefficients for polyatomic molecules. J. Chem. Phys. 1995, 103, 9347-9354.

33. Jamorski, C.; Casida, M.E.; Salahub, D.R. Dynamic polarizabilities and excitation spectra from a molecular implementation of time-dependent density-functional response theory: N2 as a case study. J. Chem. Phys. 1996, 104, 5134-5147.

34. Bauernschmitt, R.; Ahlrichs, R. Treatment of electronic excitations within the adiabatic approximation of time dependent density functional theory. Chem. Phys. Lett. 1996, 256, 454-464.

35. Casida, M.E.; Jamorski, C.; Casida, K.C.; Salahub, D.R. Molecular excitation energies to high-lying bound states from time-dependent density-functional response theory: Characterization and correction of the time-dependent local density approximation ionization threshold. J. Chem. Phys. 1998, 108, 4439-4449.

Sample Availability: Samples of compound $\left[\mathrm{Ru}\left(o-\mathrm{C}_{6} \mathrm{H}_{4}-\mathrm{py}\right)(\mathrm{MeCN})_{4}\right]^{+}$are available from the authors.

(C) 2012 by the authors; licensee MDPI, Basel, Switzerland. This article is an open access article distributed under the terms and conditions of the Creative Commons Attribution license (http://creativecommons.org/licenses/by/3.0/). 